\title{
Silicosis and Smoking: Intrinsic Phenomenon in the Respiratory System
}

\author{
Diemen Delgado Garcia ${ }^{*}$, Nayab Mahmood Sultan², Oscar Ramirez Yerba ${ }^{3}$, \\ Sofia Jimenez Castro ${ }^{4}$, Enmanuel Agila Palacios ${ }^{5}$, Ashley Delgado Cano ${ }^{1}$ \\ ${ }^{1}$ Research Institute for Safety and Health at Work, University of Guadalajara, Guadalajara, Mexico \\ ${ }^{2}$ Department of Environmental Sciences and Earth, University of Birmingham, Birmingham, UK \\ ${ }^{3}$ Occupational Medicine and the Environment, Scientific University of the South, Lima, Peru \\ ${ }^{4}$ Occupational Medicine, University of Manchester, Manchester, UK \\ ${ }^{5}$ Faculty of Security and Risks, University of the Armed Forces of Ecuador, Quito, Ecuador \\ Email: ^diemendelgadogarcia@gmail.com
}

How to cite this paper: Garcia, D., Sultan, N. M., Yerba, O. R., Castro, S. J., Palacios, E. A., \& Cano, A. D. (2018). Silicosis and Smoking: Intrinsic Phenomenon in the Respiratory System. Advances in Applied Sociology, 8, 659-667.

https://doi.org/10.4236/aasoci.2018.810039

Received: July 31, 2018

Accepted: September 27, 2018

Published: September 30, 2018

Copyright (C) 2018 by authors and Scientific Research Publishing Inc. This work is licensed under the Creative Commons Attribution International License (CC BY 4.0).

http://creativecommons.org/licenses/by/4.0/

\begin{abstract}
Purpose of the Review: Some physiopathological mechanisms that could support the relationship between tobacco and silicosis have been postulated but exact pathogenesis remains unknown. Recent Findings: Local inflammation in workers with silicosis is a complex process characterized by an infiltration of inflammatory cells in the respiratory alveolus, accompanied by an increase in the expression of cytokines, chemokines, enzymes, growth factors and adhesion molecules. Although in smokers without silicosis a similar pattern of inflammation can be observed, in workers with silicosis this process seems to be characterized by more pronounced increases in structural damage in the lungs. Altered balance of innate and adaptive immunity both play key roles in the pathogenesis of extensive fibrosis in a molecular level, influenced by individual susceptibilities and genetic traits. Conclusion: The identification of the molecular mechanism and a potential protective genotype for silicosis opens a window for the eradication of this occupational respiratory disease. This should encourage us to continue exploring and searching for the relation between the genetic polymorphism and the inorganic silica particle.
\end{abstract}

\section{Keywords}

Silicosis, Smoking, Macrophage, Apoptosis, Genotype

\section{Introduction}

The World Health Organization (WHO) considers smoking as one of the biggest 
global public health concerns with an estimated 7 million deaths worldwide-of this $6 \mathrm{~m}$ attributed to smoking and another 890,000 due to non-smokers being exposed to second-hand or passive smoke which contains over 250 which are known to be harmful and more than 50 known carcinogens (World Health Organization, 2015 \& 2017).

The relation between tobacco and silicosis is not entirely understood, although what is clear is that smoking is considered a high risk factor in the development of fibrotic as well as emphysematous lungdisease (Churg, Zhou, Preobrazhenska, Tai, Wang, \& Wright, 2009), with both active or former smokers being $60 \%$ more likely to develop silicosis (Katzenstein, Mukhopadhyay, Zanardi, \& Dexter, 2010). Some pathophysiologic mechanisms that can connect the relation between tobacco and silicosis are established. We know from current research that silicosis induces oxidative stress, increments epithelial apoptosis and alters the regulation of immunological response and the recruitment of inflammatory cells, macrophages (Rimal, Greenberg, \& Rom, 2005) and that smoking itself induces epigenetic alterations which persist even after the smoking habit has ceased. In fact, it is known that tobacco smokers show higher levels of hypermethylation, as well as of DNA-methyltransferase-1, and a reduction of the histone deacetylase 2 functions. Both changes have been associated to the fibrogenic process that occurs during the early stages of development of silicosis.

Furthermore, silicosis is typically described as a rare inflammatory/interstitial disease of the respiratory system, which causes permanent damage to the pulmonary parenchyma and the bronchial tree as consequence of an inflammatory influence caused by inhalation of respirable crystalline silica dust (Perkins et al., 2015). It is in fact a complex, multifactorial irreversible pneumoconiosis resulting from the interaction between the inhaling of an inorganic particle and a dynamic chronic inflammatory response. These inflammatory processes can ultimately lead to a structural modification of the lungs, resulting in lung fibrosis and the presence of alterations in the airflow (Naghadehi, Sereshki, \& Mohammadi, 2014). This inflammation affects all areas of the respiratory system, including the pulmonary parenchyma and both central and peripheral airways. Even though inflammation is one of the main characteristics of silicosis, the reason for this inflammatory response, the physiopathology, and progression to silicosis is still not yet fully understood. Unfortunately, the number of inflammatory mediators and their complex interrelations has made it impossible to develop a simplified pathogenic model. Due to this there are several investigations as of today that biomedical research has not been able to provide clear, decisive and accurate answers for.

Similarly, neither the exact mechanisms involved in lung damage in workers with silicosis who are also smokers nor the roles of the different inflammatory components are not completely understood. In this context, a more detailed characterization of the whole range of inflammatory responses in silicosis can pave the way for a deeper understanding the pathogenesis of the disease as a complex entity (Sultan, 2017a) especially when smoking is present. So, it is then 
necessary to recognize the role of the various cells that are involved in the inflammatory response, as well as the diverse biological causes that are part of its genesis and forms the basis of this article.

\section{Silica-Induced Cellular and Molecular Responses}

The initial inflammatory response produced in the pulmonary alveolus by the sustained, chronic inhalation of respirable-form crystalline silica (Li et al., 2017) is considered as a risk factor in the development of silicosis. This exposure is able to induce long term pathological changes in lung tissue, which could ultimately lead to the occurrence of functional alterations leading to a completed development of an occupational disease, which is asymptomatic at the beginning but could potentially leads to a massive pulmonary fibrosis (Abdelaziz, Elkashef, \& Said, 2017).

In this instance, it's necessary to point out some of the main characteristics of silicosis-related inflammation. Local inflammation in silicosis-afflicted workers is a complex process characterized by an infiltration of inflammatory cells into the pulmonary alveolus, along an increase in the production of cytokines (Skuland, 2014a, 2014b), chemokines, enzymes, growth factors and adhesion molecules. Also, some studies have demonstrated that serum elevations of biomarkers like NSE and CA125, which correlates with the severity of silicosis in some cases (Fang, Zhang, Wang, \& Zhang, 2014). At the same time, the inflammatory process after silica exposure also involve a considerable number of different cell types, including macrophages, neutrophils, eosinophils, histiocytes, epithelial cells, endothelial cells, dendritic cells, lymphocytes, fibroblasts, and pneumocytes. At the same time, localized inflammation is closely intertwined with various biological pathways, among which are an altered cellular signaling, the increase of oxidative stress, the protease-antiprotease imbalance, and the induction of apoptosis (Borges et al., 2002).

A growing body of scientific evidence suggests that local inflammation in silicosis can be modified by other factors like genetic-epigenetic mechanisms, the microbiota of the respiratory tract, age, autoimmune reactions, local hypoxia and complex cell fibroblast transformation, all altering the fine equilibrium between the anti-fibrotic and fibrotic response, necessary to keep the elasticity of lung tissue. For instance, it has been postulated that the epithelial/mesenchymal transition, a process by which the bronchial-alveolar epithelial cells become a myofibroblast, is a clue step toward the fibrotic process because it potentially alters the structure of this cell, their contraction, migration and production of extracellular matrix (Deng et al., 2016).

Based on current evidence which points to the different aspects of an inflammatory response is not only due to the exposure to silica particles, but by the physiological response is also based on individual traits and susceptibilities related with expression of specific genes during the inflammatory response (Leung, Yu, \& Chen, 2012). These genes, it has been postulated, are related in different components of the fibrotic response like over expression of inflammatory cyto- 
kines and chemokines, dissolution of extracellular matrix, wound healing, cell signaling during, antioxidant defenses and tissue remodeling (Perkins, Peeters, Wouters, Reynaert, \& Mossman, 2014).

\section{Smoking in Silicotic Lung Disease}

It has been observed in workers with silicosis that local inflammation is driven by an exaggerated or abnormal response because of the inhalation of tobacco smoke. Although in smokers without silicosis a similar pattern of inflammation can be observed, in those with silicosis the inflammation seems to be characterized by more pronounced structural damage of the respiratory system.

The molecular mechanisms underlying the exaggerated inflammatory response in silicosis cannot be fully explained, but they affect both innate and acquired immunity (Gardiner \& Mills, 2016). In case of innate response, macrophages are the main phagocytes of the respiratory system that maintain the sterility of the lower respiratory tract which are often colonized with microorganisms, mainly bacteria. In patients with silicosis, there is a marked increase (between 5 and 10 times) in the number of macrophages in the respiratory tract, the pulmonary parenchyma, in the bronchoalveolar lavage and sputum. At the same time, it is known that exposure to tobacco in workers with silicosis is associated with a deterioration in the capacity of the macrophages to eliminate respiratory pathogens and apoptotic cells (Delgado, Parra, \& Capelozzi, 2006). This fact has pointed out the vital role of alveolar macrophages in the preventing a persistent colonization so smoking could be an important cause of defective phagocytosis and elimination of bacteria which ultimately leads to abnormal colonization and a vicious circle between inflammation and infection.

There is also clinical evidence that would indicate that the activation of alveolar macrophages by silica produces a rapid and sustained inflammation that is characterized by the generation of protein 1 Monocyte chemotactic MCP-1, which induces fibrosis. This finding provides a new perspective on the potential of MCP-1 in the development of new therapeutic strategies against silicosis (Liu et al., 2015). In another aspect, the increase in the number of macrophages in the lungs of smokers and in workers with silicosis are caused by the increased recruitment of monocytes from circulation as a response to monocyte-selective chemokines (Liu et al., 2018) of CKLF1, however, despite this increase in the number of macrophages in the respiratory tract of silicosis-suffering smokers, persistent colonization is also a common finding. This suggests that these cells have a reduced ability to phagocytose bacteria found in the lung, mainly Haemophilusinfuenzae and Streptococcus pneumoniae, in comparison to healthy subjects.

In the case of acquired immunity in silicosis it has been reported an abnormal response to tobacco smoke from Regulatory $\mathrm{T}$ Cells and an increase in $\mathrm{T}$ helper type 1 (Th1) as well as participation of Th17 cell responses which are characteristic of human emphysema. For instance, animal experimental models have 
shown that Th1 and Th17 cytokines and elastases response, could be induced by cigarette smoke related emphysema (Shan et al., 2012). Similarly, interferon- $\gamma$ (IFN- $\gamma$ ) and interleuking-17A (IL17A) produced by both $\mathrm{CD} 4^{+}$and $\gamma \delta \mathrm{T}$ cells, were also increased.

The effects of quitting smoking on local inflammation in workers with silicosis are still debated. However, there is evidence that stopping smoking at an early stage effectively reduces the inflammatory reaction in silicosis, suggesting value of stopping smoking at the onset of diagnosis of the disease. It is probable that epithelial cells and macrophages are the first cell types to encounter tobacco smoke and, therefore, the first to act and trigger the inflammatory response.

The involvement of the alveolar epithelium in the pathogenesis of silicosis associated with tobacco smoke has three main defenses. First, as a physical barrier. The epithelial cells of the respiratory tract are important in the defense of the lungs, with the production of mucus from goblet cells, dendritic cells (Nicod, 2005), and the secretion of antioxidants, anti-proteases and defensins, as well as the constant ciliary movement. It is possible that tobacco smoke and other harmful agents can alter these responses of the epithelium of the respiratory tract, contributing to the injury produced and increasing the susceptibility to infection. Second, by the production of inflammatory mediators upon contact, epithelial cells are activated by tobacco smoke and begin to produce inflammatory mediators, including TNF- $\alpha$, IL-1 $\beta$, IL-6 (Chan et al., 2018; Yucesoy et al., 2001), a stimulation factor of granulocyte colonies activated by chemokines (Adage et al., 2015) and macrophages (gM-CsF), all of which play a crucial role in the inflammation and alteration of lung tissue. In addition, epithelial cells of the small airways can also be an important source of transforming growth factor beta which would later induce local fibrosis ( $\mathrm{Li}$ et al., 2017). Third, pneumocytes are also susceptible, and alterations can occur at this level. In this sense, the presence of vascular endothelial growth factor (VEgF) seems to be necessary to maintain the integrity of the alveolar cell (Truong, Dhapare, Desai, Voelkel, \& Sakagami, 2017), resulting in emphysema as a secondary disorder (Zhang et al., 2016).

So it seems that accumulation of necrotic material in the lung, contributes to perpetuates the inflammation; At the same time, an exaggerated innate immune response, increase in the cells of the adaptive immune system of both lymphocytes T as of CD8 (Emad \& Emad, 2007) in patients with both conditions. This increase is relevant for two reasons. First, because it seems to be one of the main differences in the inflammatory infiltrate of non-smoking workers with silicosis; And second, because there is a correlation between the number of $\mathrm{T}$ cells, the amount of alveolar destruction and the severity of airflow obstruction in workers with silicosis who smoke (Delgado, Aguilera, Delgado, \& Rug, 2012).

\section{Conclusion}

Our current knowledge of the biological mechanisms underlying the inflammation of silicosis has increased significantly in recent years but remains far from complete before focusing on the identification of new potential inflammatory 
biomarkers. It would require a deeper knowledge of the basic mechanisms of inflammation that occurs in silicosis.

Direct comparisons of different studies measuring inflammation biomarkers in silicosis and smoking (Baumgartner, Samet, Stidley, Colby, \& Waldron, 1997) should be made with caution due to a series of possible confounding factors such as compartmentalization or interaction with the various biological pathways and cell types involved.

Although the studies available to date have focused specifically on a limited number of candidate biomarkers for inflammation, future research should be designed with a broader approach that covers the major inflammatory disturbances that occur in silicosis. This identification of biomarkers capable of tracking both local and systemic inflammation in silicosis can be achieved through rigorous and well-designed studies.

The identification of potential factors known to be sources of inflammation and their correlation with an increased risk of silicosis will provide mechanistic clues to this research question as knowledge advances, we will be able to better understand the development and continuation of this disease, which will allow us to identify new therapeutic targets.

Efforts to identify the genetic determinants of silicosis have evolved as available technologies have changed, using two different and complementary approaches (Wang, Lan, Yang, De, \& Kuang, 2012). On the one hand, the analysis of candidate genes starts from a hypothesis which connects a specific gene with the disease and tries to assess whether this gene-silicosis relationship exists, and on the other hand high performance technology such as DNA microarrays allow testing up to one million single nucleotide polymorphisms (SNP) at a time in each case (Shan et al., 2012; Yuan et al., 2017).

This approach does not relate to any hypothesis and therefore it is a technique that allows opening new research channels. In the same way, the identification of the protective genotype (Fang, Fan, \& Shen, 2011) for silicosis opens a window for the eradication of this historical occupational respiratory disease from the world which still claims annually tens of thousands of lives worldwide (Sultan, 2017b) which should encourage us to continue exploring in the search for the relationship between the genetic polymorphism of the IL-4-33 site and silicosis, as a protective genotype for workers who are exposed to the inorganic silica particle.

\section{Acknowledgements}

The authors dedicate this work to Eustorgio Delgado Palma and Bethy Garcia Pinoargote as a source of inspiration to the new generations of researchers in public health in the world.

\section{Conflicts of Interest}

The authors declare no conflicts of interest regarding the publication of this paper. 


\section{References}

Abdelaziz, R. R., Elkashef, W. F., \& Said, E. (2017). Tadalafil Reduces Airway Hyperactivity and Protects against Lung and Respiratory Airways Dysfunction in a Rat Model of Silicosis. International Immunopharmacology, 40, 530-541. https://doi.org/10.1016/j.intimp.2016.10.007

Adage, T. et al. (2015). Targeting Glycosaminoglycans in the Lung by an Engineered CXCL8 as a Novel Therapeutic Approach to Lung Inflammation. European Journal of Pharmacology, 748, 83-92. https://doi.org/10.1016/j.ejphar.2014.12.019

Baumgartner, K. B., Samet, J. M., Stidley, C. A., Colby, T. V., \& Waldron, J. A. (1997). Cigarette Smoking: A Risk Factor for Idiopathic Pulmonary Fibrosis. American Journal of Respiratory and Critical Care Medicine, 155, 242-248. https://doi.org/10.1164/ajrccm.155.1.9001319

Borges, V. M. et al. (2002). Apoptosis Underlies Immunopathogenic Mechanisms in Acute Silicosis. American Journal of Respiratory Cell and Molecular Biology, 27, 78-84. https://www.ncbi.nlm.nih.gov/pubmed/12091249 https://doi.org/10.1165/ajrcmb.27.1.4717

Chan, J. W. et al. (2018). RNA-Seq Revealed ATF3-Regulated Inflammation Induced by Silica. Toxicology, 393, 34-41. https://doi.org/10.1016/j.tox.2017.11.001

Churg, A., Zhou, S., Preobrazhenska, O., Tai, H., Wang, R., \& Wright, J. L. (2009). Expression of Profibrotic Mediators in Small Airways versus Parenchyma after Cigarette Smoke Exposure. American Journal of Respiratory Cell and Molecular Biology, 40, 268-276. https://www.ncbi.nlm.nih.gov/pubmed/18723441 https://doi.org/10.1165/rcmb.2007-0367OC

Delgado, D., Aguilera, M. A., Delgado, F., \& Rug, A. (2012). The Experience of Miners Relocated to Alternative Positions due to Silicosis in the Andean of CODELCO, Chile, 2010. Safety and Health at Work, 3, 140-145. https://doi.org/10.5491/SHAW.2012.3.2.140

Delgado, L., Parra, E. R., \& Capelozzi, V. L. (2006). Apoptosis and Extracellular Matrix Remodeling in Human Silicosis. Histopathology, 49, 283-289.

https://www.ncbi.nlm.nih.gov/pubmed/16918975 https://doi.org/10.1111/j.1365-2559.2006.02477.x

Deng, H. et al. (2016). Protective Effect of Ac-SDKP on Alveolar Epithelial Cells through Inhibition of EMT via TGF- $\beta 1 /$ ROCK1 Pathway in Silicosis in Rat. Toxicology and Applied Pharmacology, 294, 1-10. https://doi.org/10.1016/j.taap.2016.01.010

Emad, A., \& Emad, Y. (2007). Increased in CD8 T lymphocytes in the BAL Fluid of Patients with Sulfur Mustard Gas-Induced Pulmonary Fibrosis. Respiratory Medicine, 101, 786-792. https://doi.org/10.1016/j.rmed.2006.08.003

Fang, G. F., Fan, X. Y., \& Shen, F. H. (2011). The Relationship between Polymorphisms of Interleukin-4 Gene and Silicosis. Biomedical and Environmental Sciences, 24, 678-682. https://www.ncbi.nlm.nih.gov/pubmed/22365405

Fang, S. C., Zhang, H. T., Wang, C. Y., \& Zhang, Y. M. (2014). Serum CA125 and NSE: Biomarkers of Disease Severity in Patients with Silicosis. Clinica Chimica Acta, 433, 123-127. https://doi.org/10.1016/j.cca.2014.03.005

Gardiner, C. M., \& Mills, K. H. (2016). The Cells That Mediate Innate Immune Memory and Their Functional Significance in Inflammatory and Infectious Diseases. Seminars in Immunology, 28, 343-350. https://doi.org/10.1016/j.smim.2016.03.001

Katzenstein, A. L., Mukhopadhyay, S., Zanardi, C., \& Dexter, E. (2010). Clinically Occult Interstitial Fibrosis in Smokers: Classification and Significance of a Surprisingly Common Finding in Lobectomy Specimens. Human Pathology, 41, 316-325. 
https://doi.org/10.1016/j.humpath.2009.09.003

Leung, C. C., Yu, I. T., \& Chen, W. (2012). Silicosis. The Lancet, 379, 2008-2018. https://doi.org/10.1016/S0140-6736(12)60235-9

Li, J. et al. (2017). Crystalline Silica Promotes Rat Fibrocyte Differentiation in Vitro, and Fibrocytes Participate in Silicosis in Vivo. Biomedical and Environmental Sciences, 30, 649-660.

Li, X. et al. (2017). Bone Marrow Mesenchymal Stem Cells Attenuate Silica-Induced Pulmonary Fibrosis via Paracrine Mechanisms. Toxicology Letters, 27, 96-107. https://doi.org/10.1016/j.toxlet.2017.02.016

Liu, D. D. et al. (2018). Progress in Pharmacological Research of Chemokine like Factor 1 (CKLF1). Cytokine, 102, 41-50. https://doi.org/10.1016/j.cyto.2017.12.002

Liu, X. et al. (2015). Role of Human Pulmonary Fibroblast-Derived MCP-1 in Cell Activation and Migration in Experimental Silicosis. Toxicology and Applied Pharmacology, 288, 152-160. https://doi.org/10.1016/j.taap.2015.07.002

Naghadehi, M. Z., Sereshki, F., \& Mohammadi, F. (2014). Pathological Study of the Prevalence of Silicosis among Coal Miners in Iran: A Case History. Atmospheric Environment, 83, 1-5. https://doi.org/10.1016/j.atmosenv.2013.10.053

Nicod, L. P. (2005). Lung Defenses: An Overview. European Respiratory, 14, 45-50. http://err.ersjournals.com/content/14/95/45

Perkins, T. N. et al. (2015). Indications for Distinct Pathogenic Mechanisms of Asbestos and Silica through Gene Expression Profiling of the Response of Lung Epithelial Cells. Human Molecular Genetics, 24, 1374-1389. https://doi.org/10.1093/hmg/ddu551 https://www.ncbi.nlm.nih.gov/pubmed/25351596

Perkins, T. N., Peeters, P. M., Wouters, E. F. M., Reynaert, N. L., \& Mossman, B. T. (2014). Pathogenesis and Mechanisms of Asbestosis and Silicosis. In L. M. McManus, \& R. N. Mitchell (Eds.), Pathobiology of Human Disease (pp. 2654-2664). Cambridge, MA: Academic Press. https://doi.org/10.1016/B978-0-12-386456-7.05308-9

Rimal, B., Greenberg, A. K., \& Rom, W. N. (2005). Basic Pathogenetic Mechanisms in Silicosis: Current Understanding. Current Opinion in Pulmonary Medicine, 11, 169-173. https://www.ncbi.nlm.nih.gov/pubmed/15699791 https://doi.org/10.1097/01.mcp.0000152998.11335.24

Shan, M. et al. (2012). Cigarette Smoke Induction of Osteopontin (SPP1) Mediates $\mathrm{T}(\mathrm{H}) 17$ Inflammation in Human and Experimental Emphysema. Science Translational Medicine, 4, 117-119. https://www.ncbi.nlm.nih.gov/pubmed/22261033 https://doi.org/10.1126/scitranslmed.3003041

Skuland, T., Ovrevik, J., Lag, M., \& Refsnes, M. (2014a). Role of Size and Surface Area for Pro-Inflammatory Responses to Silica Nanoparticles in Epithelial Lung Cells: Importance of Exposure Conditions. Toxicology in Vitro, 28, 146-155. https://doi.org/10.1016/j.tiv.2013.10.018

Skuland, T., Ovrevik, J., Lag, M., Schwarze, P., \& Refsnes, M. (2014b). Silica Nanoparticles Induce Cytokine Responses in Lung Epithelial Cells through Activation of a p38/TACE/TGF- $\alpha$ /EGFR-Pathway and NF- $\kappa B$ Signaling. Toxicology and Applied Pharmacology, 279, 76-86.

Sultan, N. (2017a). The Silent Killer. Solids and Bulk Handling, 3, 17-18. http://www.academia.edu/32341180/The_Silent_Killer

Sultan, N. (2017b). The Silica Dust Hazard-Why I Should Be Concerned? Canadian Design and Construction Report, 8, 12-17. 
http://www.academia.edu/31084926/The_silica_dust_hazard_why_I_should_be_conce rned

Truong, T. M., Li, H., Dhapare, S., Desai, U. R., Voelkel, N. F., \& Sakagami, M. (2017). Sulfated Dehydropolymer of Caffeic Acid: In Vitro Anti-Lung Cell Death Activity and in Vivo Intervention in Emphysema Induced by VEGF Receptor Blockade. Pulmonary Pharmacology \&Therapeutics, 45, 181-190. https://doi.org/10.1016/j.pupt.2017.06.007

Wang, Y. W., Lan, J. Y., Yang, L. Y., De J. W., \& Kuang, J. (2012). TNF- $\alpha$ and IL-1RA Polymorphisms and Silicosis Susceptibility in Chinese Workers Exposed to Silica Particles: A Case-Control Study. Biomedical and Environmental Sciences, 25, 517-525.

World Health Organization (2015). Global Report on the Trends in Prevalence of Tobacco Smoking.

http://www.who.int/tobacco/publications/surveillance/trends-tobacco-smoking-second -edition/en/

World Health Organization (2017). Report on the Global Tobacco Epidemic. http://www.who.int/tobacco/global_report/en/

Yuan, J. et al. (2017). Polymorphisms in Autophagy Related Genes and the Coal Workers' Pneumoconiosis in a Chinese Population. Gene, 632, 36-42.

https://doi.org/10.1016/j.gene.2017.08.017

Yucesoy, B. et al. (2001). Association of Tumor Necrosis Factor-Alpha and Interleukin-1 Gene Polymorphisms with Silicosis. Toxicology and Applied Pharmacology, 172, 75-82. https://doi.org/10.1006/taap.2001.9124

Zhang, L. et al. (2016). Combined Pulmonary Fibrosis and Emphysema: A Retrospective Analysis of Clinical Characteristics, Treatment and Prognosis. BMC Pulmonary Medicine, 16, 137. https://www.ncbi.nlm.nih.gov/pubmed/27809901

https://doi.org/10.1186/s12890-016-0300-7 